E. Gutkin

Nagoya Math. J.

Vol. 149 (1998), 93-116

\title{
GREEN'S FUNCTIONS OF FREE PRODUCTS OF OPERATORS, WITH APPLICATIONS TO GRAPH SPECTRA AND TO RANDOM WALKS
}

\author{
EUGENE GUTKIN ${ }^{1}$
}

\begin{abstract}
We systematically develop an algebraic technique of free products of operators and their Green's functions. We apply this framework to obtain, in a simple and uniform fashion, several results on the spectra of graph Laplaceans and random walks.
\end{abstract}

\section{Introduction}

Let $X, Y$ be discrete groups, and let $X * Y$ be their free product. Let $A, B$ be bounded operators on $\ell^{2}(X), \ell^{2}(Y)$ respectively. Their free product, $A * B$, is an operator on $\ell^{2}(X * Y)$. The pairing $A, B \mapsto A * B$ is a natural operation. If $A$ and $B$ are convolution operators, then $A * B$ is a convolution operator. Let $S$ and $T$ be generating sets of the groups $X$ and $Y$, and let $A$ and $B$ be the corresponding incidence operators. Then $A * B$ is the incidence operator on $X * Y$ corresponding to the generating set $S \cup T$.

The Green's functions of $A, B$ and $A * B$ satisfy a system of algebraic equations. If $A$ and $B$ are selfadjoint convolution operators, the system yields considerable information about the spectrum of $A * B$ [7]. For instance, $A * B$ has no singular continuous spectrum. If $\sigma_{p}(A)$ and $\sigma_{p}(B)$ are the point spectra of $A$ and $B$, then $\sigma_{p}(A * B) \subset \sigma_{p}(A)+\sigma_{p}(B)$. The (absolutely) continuous spectrum of $A * B$ is a union of a finite number of intervals.

The present work is rooted in the observation that the group structure is largely irrelevant for these results. Let $X$ and $Y$ be arbitrary countable sets, each with a marked element, the root. We say that $X, Y$ are rooted sets. Their free product, $X * Y$, is a rooted set, as well. Let $A$ and $B$ be operators on $\ell^{2}(X)$ and $\ell^{2}(Y)$, respectively. We define the free product operator, $A * B$, on $\ell^{2}(X * Y)$. If $X, Y$ are groups, and the roots are the

Received July 22, 1996.

${ }^{1}$ Partially supported by NSF Grants DMS-9013220, DMS-9400295. 
respective identity elements, then $X * Y$ is the free product of groups, and $A * B$ coincides with the free product operator in the group sense.

Our construction extends to an arbitrary number of factors, yielding the free products $\mathcal{A}=*_{i=1}^{n} A_{i}$. Let $G_{i}, 1 \leq i \leq n$, and $\mathcal{G}$ be the Green's functions of $A_{i}, 1 \leq i \leq n$, and $\mathcal{A}$, respectively. We obtain a system of $n+1$ algebraic equations on $G_{i}$, and $\mathcal{G}$ (see Theorem 1 and Corollaries $1-3$, especially Corollary 3 ). This system is a source of information about $\mathcal{G}$. If the operators $A_{i}$ are selfadjoint and invariant with respect to groups $\Gamma_{i}$, transitively acting on $X_{i}, 1 \leq i \leq n$, then $\mathcal{G}$ determines the spectrum of $\mathcal{A}$.

The system of equations of Corollary 3 can be explicitly solved only in very special cases. In Section 3 we investigate two such cases. In Section 3.1 we consider the free products $A * B$ where each of the operators $A, B$ has two distinct eigenvalues (with arbitrary multiplicities). By Proposition 1 , the Green's function of $A * B$ is single-valued on a double covering of C, given by $\mathcal{R}=\left\{(t, w): w^{2}=R(t)\right\}$, where $R(t)$ is a quartic polynomial. In particular, $\mathcal{R}$ is an elliptic curve. In Section 3.2 we obtain the Green's function of any $*^{n} A$, if $A$ has two eigenvalues (Proposition 5).

Explicit expressions for the Green's functions yield the spectra of the corresponding free product operators (Proposition 2, Theorems 2 and 3). The absolutely continuous spectrum consists of one or two intervals, whose endpoints are algebraic functions of the parameters involved (Theorems 2 and 3). The point spectrum is more involved. We completely determine the point spectra of $*^{n} A$ (Theorem 3 ), and get a good bound on the point spectra of $A * B$ (Proposition 2). We will report more comprehensively on this subject elsewhere [5].

There has been much interest in the spectra of graph Laplaceans, and, more generally, in the random walks on graphs [12]. In particular, there is considerable literature on random walks on free group products, and the spectra of related graphs (see the references in [12]). Several seemingly unrelated techniques have been used to calculate explicitly these spectra in special cases $[1,2,3,6,7,8,10,11]$.

The approach developed here allows to obtain these results in a simple and uniform way. Let $K_{n}$ be the complete graph on $n+1$ vertices. In Section 4.1 we calculate the spectra of $K_{m} * K_{n}$ and $*^{n} K_{r}$ for arbitrary $m, n, r$ (Theorems 4 and 5). In Section 4.2 we determine the spectra of the product random walks on $K_{m} * K_{n}$, and of the simple random walks on $*^{n} K_{r}$, for arbitrary $m, n, r$ (Theorems 6 and 7 ). We use the results of 
Section 3, and exploit the elementary fact that the spectrum of a complete graph consists of two numbers.

We thank B. Simon for fruitful discussions and for providing the reference [9]. We also thank the anonimous referee for asking the right questions.

\section{$\S 1$. Preliminaries}

A rooted set, $(X, e)$, is a countable (at most) set with a distinguished element, the root. We denote by $\ell^{2}(X)$ the Hilbert space of square summable functions on $X$. There is a natural correspondence between operators and kernels:

$$
(A f)(x)=\sum_{y \in X} A(x, y) f(y) .
$$

If $A(x, y) \neq 0$, we write $x \leadsto y$. If $x \sim y$ and $y \leadsto x$, we say that $x, y$ are neighbors, and write $x \sim y$.

Condition 1. i) For all $x \in X$, we have $|\{y: x \sim y\}|=q(x) \leq q(A)=$ $q<\infty$; ii) $\max _{x, y}|A(x, y)|=p(A)=p<\infty$.

ExAMPLE. The set $X$ is a regular graph of degree $q$ (i.e., $q$ neighbors of a vertex), and $A$ is the incidence operator: $A(x, y)=A(y, x)=1$ if $x$ and $y$ are connected by an edge, and $A(x, y)=0$ otherwise.

In what follows we assume Condition 1 (unless stated otherwise). Then $A$ is bounded, $\|A\| \leq p q$ (by Schwarz' inequality). Set

$$
A^{n}(x, y)=\sum_{v_{1}, \ldots, v_{n-1}} A\left(x, v_{n-1}\right) \cdots A\left(v_{1}, y\right), n>0, A^{0}(x, y)=\delta_{x, y}
$$

and define

$$
F_{x, y}(t)=\sum_{n \geq 0} A^{n}(x, y) t^{n} .
$$

By Condition 1, the series converges for sufficiently small $|t|$, and $F_{x, y}(t)=$ $(1-t A)^{-1}(x, y)$. We set $F_{x}(t)=F_{x, x}(t)$, and call it the return function for $x$. The first return function, $f_{x}(t)$, is defined by

$$
f_{x}(t)=\sum_{n \geq 1} t^{n}\left(\sum_{v_{\imath} \neq x} A\left(x, v_{1}\right) \cdots A\left(v_{n-1}, x\right)\right)
$$


and we have

$$
F_{x}(t)=\left(1-f_{x}(t)\right)^{-1} .
$$

We denote by $G_{x, y}(t)$ the kernel of the resolvent, $R(t)=(t-A)^{-1}$. For $|t|$ sufficiently large

$$
G_{x, y}(t)=\frac{1}{t} F_{x, y}\left(t^{-1}\right) .
$$

We set $G_{x}(t)=G_{x, x}(t)$. In what follows we assume that $A(x, y)=A(y, x) \in$ $\mathbf{R}$. Then $A$ is a bounded selfadjoint operator. The formulas above provide a connection between the return functions and the spectrum of $A$.

EXAmple. For $|X|=n<\infty$, let $\lambda_{1} \leq \cdots \leq \lambda_{n}$ be the eigenvalues of $A$, and let $\phi_{i}(x)$ be the corresponding orthonormal (real) eigenfunctions. Then

$$
G_{x, y}(t)=\sum_{i} \frac{\phi_{i}(x) \phi_{i}(y)}{t-\lambda_{i}}
$$

is rational, with the simple poles at the eigenvalues of $A$.

If $A$ is the incidence operator of a graph $X$, the return functions have a geometric interpretation: $A^{n}(x, y)$ is the number of walks on $X$ of length $n$ starting at $y$ and ending at $x$. Thus $F_{x}(t)$ is the counting function for the closed walks starting at $x$, and $f_{x}(t)$ counts the closed walks that do not come back prematurely.

Definition 1. Let $A$ be an operator on $\ell^{2}(X)$. We say that $A$ is invariant if there is a group, $\Gamma$, acting transitively on $X$, and $A(g x, g y)=$ $A(x, y)$ for $g \in \Gamma$. If $X$ is a graph, and its incidence operator is invariant, we say that the graph is symmetric.

If $A$ is invariant, then $F_{x}(t), f_{x}(t)$, and $G_{x}(t)$ do not depend on $x$. Let $|X|<\infty$, and let $A$ be an invariant operator. In the preceding example, for any $x$

$$
G_{x}(t)=\sum_{i} \frac{\phi_{i}^{2}(x)}{t-\lambda_{i}}
$$

Denote by $\Lambda$ the set of eigenvalues of $A$, and let $m(\lambda) \geq 1$ be the multiplicity, $\sum_{\lambda} m(\lambda)=|X|$. Since $\sum_{x} \phi_{i}^{2}(x)=1$ for any $i$, we have

(6) $\quad G_{x}(t)=\frac{1}{|X|} \sum_{\lambda \in \Lambda} \frac{m(\lambda)}{t-\lambda}=G(t), \quad F_{x}(t) \doteq \frac{1}{|X|} \sum_{\lambda \in \Lambda} \frac{m(\lambda)}{1-\lambda t}=F(t)$. 
Let the notation be as above. If $G_{x}(t)$ does not depend on $x \in X$, or if $x$ is a distinguished element (see Section 2 below), we say that $G_{x}(t)=G(t)$ is the Green's function.

\section{$\S 2$. Free Products of Operators and Green's Functions}

Let $\left(X_{i}, e_{i}\right), 1 \leq i \leq n, n \geq 2$, be arbitrary rooted sets. Identifying the roots in the union, $\cup_{i} X_{i}$, we obtain the bouquet, $\left(\#_{i} X_{i}, e\right)$, of the sets $X_{i}$, where the root $e$ is the image of the points $e_{i}$. The notation $\left(\#_{i \in I} X_{i}, e\right)$, where $I \subset\{1, \ldots, n\}$, is self-explanatory. We will now define the free product, $*_{i=1}^{n}\left(X_{i}, e_{i}\right)=(\mathcal{X}, e)$.

Set $\tilde{X}_{i}=X_{i} \backslash\left\{e_{i}\right\}$, and $\mathcal{X}_{1}=\#_{i=1}^{n} X_{i}$. Then $\mathcal{X}_{1}=\{e\} \cup \tilde{X}_{1} \cup \cdots \cup \tilde{X}_{n}$, a disjoint union. Let $x \in \mathcal{X}_{1}, x \neq e$. There is a unique $i \in\{1, \ldots, N\}$, such that $x \in \tilde{X}_{i}$. We identify $x$ with the root of the bouquet $\#_{j \neq i} X_{j}$. After having done this for all $x \in \mathcal{X}_{1}$, we obtain a new rooted set, $\mathcal{X}_{2}$, where $e \in \mathcal{X}_{1} \subset \mathcal{X}_{2}$. Continuing this process indefinitely, we obtain the increasing tower $\{e\} \subset \mathcal{X}_{1} \subset \mathcal{X}_{2} \subset \cdots \subset \mathcal{X}_{m} \subset \cdots$. The union, $\mathcal{X}=\cup_{k=0}^{\infty} \mathcal{X}_{k}$, is the free product of $X_{i}, 1 \leq i \leq n$. For any point $(x) \in \mathcal{X},(x) \neq e$, there is a unique sequence, $i_{1}, \ldots, i_{m}$ of indices $i_{k} \in\{1, \ldots, n\}, i_{k} \neq i_{k-1}$ for all $k$, and for each $i_{k}$ there is a unique $x_{i_{k}} \in \tilde{X}_{i_{k}}$ so that $(x)$ is coded by the sequence $\left(x_{i_{1}}, \ldots, x_{i_{m}}\right)$. The correspondence between $\mathcal{X}$ and the set of such sequences is one-to-one. We think of elements $(x) \in \mathcal{X}$ as words, and of $m=|(x)|$ as the "length" of $(x)$. Then $\mathcal{X}_{m}=\{(x):|(x)| \leq m\}$, and $|(x)|=0$ if and only if $(x)=e$.

The construction of $*_{i=1}^{n} X_{i}$ does require the sets $X_{i}$ to be rooted, but nothing else. If each $X_{i}$ is the vertex set of a graph with a distinguished vertex, $e_{i}$, then $*_{i=1}^{n} X_{i}$ is the free product of the graphs $X_{i}, 1 \leq i \leq n$. If each $X_{i}$ is a group, and $e_{i} \in X_{i}$ is the identity, then $*_{i=1}^{n} X_{i}$ is the free product of the groups $X_{i}, 1 \leq i \leq n$, and $e$ is the identity element.

Let the notation be as above, and let $A_{i}(x, y)$ be operators on $\ell^{2}\left(X_{i}\right)$, $1 \leq i \leq n$. We will define operators $\mathcal{A}_{i}$ on $\ell^{2}(\mathcal{X})$ via their kernels $\mathcal{A}_{i}((x),(y))$. Let, for simplicity of notation, $i=1$. Let $(x)=x_{i_{1}} \cdots x_{i_{k}}$ and $(y)=$ $y_{j_{1}} \cdots y_{j_{l}}$ be in $\mathcal{X}$. If $k=0$ then $(x)=e$, and we set $\mathcal{A}_{1}(e,(y))=A_{1}\left(e_{1}, y_{1}\right)$, if $l=1$ and $j_{1}=1$, and $\mathcal{A}_{1}(e,(y))=0$ otherwise. For $k>0$ we set $\mathcal{A}_{1}((x),(y))=A_{1}\left(x_{i_{k}}, y_{j_{k}}\right)$, if $l=k, x_{i_{1}} \cdots x_{i_{k-1}}=y_{j_{1}} \cdots y_{j_{k-1}}$, and $i_{k}=$ $j_{k}=1$. Otherwise $\mathcal{A}_{1}((x),(y))=0$. If all of $A_{i}$ satisfy Condition 1 , then $\mathcal{A}_{i}$ satisfy it as well, and $p\left(\mathcal{A}_{i}\right)=p\left(A_{i}\right), q\left(\mathcal{A}_{i}\right)=q\left(A_{i}\right)$.

Definition 2. Let the notation be as above. The operator $\sum_{i=1}^{n} \mathcal{A}_{i}$ on 
$\ell^{2}\left(*_{i=1}^{n} X_{i}\right)$ is the free product of the operators $A_{1}, \ldots, A_{n}$. We denote it by $\mathcal{A}=*_{i=1}^{n} A_{i}$.

ExAmples. 1. Let $X_{i}, 1 \leq i \leq n$, be rooted graphs, and let $A_{i}$ be the incidence operator of $X_{i}$. Then $*_{i=1}^{n} A_{i}$ is the incidence operator of the graph $*_{i=1}^{n} X_{i}$. 2. Let $A_{i}$ be the transition operator for a random walk on $X_{i}, 1 \leq i \leq n$. Let $\mu_{i} \geq 0, \sum_{i=1}^{n} \mu_{i}=1$. The operator $*_{i=1}^{n} \mu_{i} A_{i}$ is the transition operator for a random walk on $*_{i=1}^{n} X_{i}$.

For $i \in\{1, \ldots, n\}$ we denote by $\mathcal{X}^{(i)} \subset \mathcal{X}$ the set of words $(x)=$ $x_{i_{1}} \cdots x_{i_{k}}$ such that $i_{1}=i$, and, by convention, set $e \in \mathcal{X}^{(i)}$. Then $\mathcal{X}^{(i)}$ are rooted sets, and $\mathcal{X}=\#_{i=1}^{n} \mathcal{X}^{(i)}$. Set $\mathcal{Y}^{(i)}=\#_{j \neq i} \mathcal{X}^{(j)}$.

In what follows we consider various rooted sets. The root will always be clear from the context, and we suppress it from notation. For $1 \leq i \leq n$ we denote by $\left(f_{i}(z)\right) F_{i}(z), G_{i}(z)$ the (first) return function and the Green's function for $A_{i}$, and by $\left(\phi_{i}(z)\right) \Phi_{i}(z)$ the (first) return function for $\mathcal{A}_{i}$ on $\mathcal{X}^{(i)}$. In the formula below the "hat" above a symbol means the symbol is deleted.

THEOREM 1. Let the setting be as above. Then, for $1 \leq i \leq n$,

$$
\begin{gathered}
F_{i}\left(\frac{z}{1-\phi_{1}(z)-\cdots-\hat{\phi}_{i}-\cdots-\phi_{n}(z)}\right) \\
=\frac{1-\phi_{1}(z)-\cdots-\hat{\phi}_{i}-\cdots-\phi_{n}(z)}{1-\sum_{k=1}^{n} \phi_{k}(z)} .
\end{gathered}
$$

Proof. We will use the language of graphs in the argument, linking the vertices $x, y$ whenever $C(x, y) \neq 0$, for a suitable kernel. Set, for simplicity of notation, $i=1$, and compute the series $\phi_{1}(z)$. Let $\Gamma$ be any loop contributing to $\phi_{1}(z)$. The first move of $\Gamma$ is from $e$ to some $x_{1} \in X_{1}$. At $x_{1}$ there is a subset of $\mathcal{X}$, isomorphic to $\mathcal{Y}^{(1)}$, "attached" by its root to $x_{1}$. From $x_{1}$ the "walk" goes into $\mathcal{Y}^{(1)}$, and makes a loop, $\beta_{1}$, in $\mathcal{Y}^{(1)}$, which ends upon return to $x_{1}$ for the last time. After that $\Gamma$ moves to another vertex, $x_{2} \in X_{1}$. Repeating this construction, we obtain a unique decomposition:

$$
\Gamma: e \rightarrow x_{1}, \beta_{1}, x_{1} \rightarrow x_{2}, \beta_{2}, \ldots, x_{k-1}, \beta_{k-1}, x_{k-1} \rightarrow e .
$$

The points $x_{1}, \ldots, x_{k-1}$ are in $X_{1}$, and are all different from $e$. Thus $e \rightarrow$ $x_{1} \rightarrow \cdots \rightarrow x_{k-1} \rightarrow e$ is a first return loop, $\gamma$, in $X_{1}$, and $\beta_{1}, \ldots, \beta_{k-1}$ are (rooted) closed walks in $\mathcal{Y}^{(1)}$. The contribution of $\Gamma$ to $\phi_{1}(z)$ factorizes in 
an obvious way. To write it down, we use the general notation $h(z \mid \alpha)$ for the contribution of $\alpha$ to a counting function, $h(z)$. Denote by $\left(\psi_{i}(z)\right) \Psi_{i}(z)$ the (first) return function for the operator $\mathcal{A}$ restricted to $\mathcal{Y}^{(i)}$. Then

$$
\phi_{1}(z \mid \Gamma)=f_{1}(z \mid \gamma) \prod_{j=1}^{k-1} \Psi_{1}\left(z \mid \beta_{j}\right)
$$

The correspondence $\Gamma=\left(\gamma ; \beta_{1} \ldots, \beta_{k-1}\right)$ is one-to-one. Fixing $\gamma$ and summing up over all $\beta_{1} \ldots, \beta_{k-1}$, we obtain an expression for the contribution to $\phi_{1}(z)$ from all loops $\Gamma$ corresponding to the same $\gamma=\bar{\Gamma},|\gamma|=k>0$ :

$$
\sum_{\bar{\Gamma}=\gamma} \phi_{1}(z \mid \Gamma)=f_{1}(z \mid \gamma) \Psi_{1}(z)^{k-1}=f_{1}\left(z \Psi_{1}(z) \mid \gamma\right) / \Psi_{1}(z)
$$

Since $\mathcal{Y}^{(1)}=\#_{i \neq 1} \mathcal{X}^{(i)}$, we have $\Psi_{1}(z)=\left(1-\hat{\phi}_{1}(z)-\phi_{2}(z) \cdots-\phi_{n}(z)\right)^{-1}$. Substituting this into eq. (8), summing up over all $\gamma$, and replacing ' ${ }^{\prime}$ ' with an arbitrary $i$ :

(9) $\phi_{i}(z) /\left[1-\sum_{j \neq i} \phi_{j}(z)\right]=f_{i}\left(\frac{z}{1-\phi_{1}(z)-\cdots-\hat{\phi}_{i}-\cdots-\phi_{n}(z)}\right)$.

Expressing $f_{i}$ in terms of $F_{i}$, by eq. (3), we obtain the claim.

Remark. The argument above does not use all of our assumptions on $X_{i}$ and $A_{i}$, only that $q_{i}(x)$ is finite for any $x \in X_{i}$.

We denote by $\mathcal{G}(z)$ and $\mathcal{F}(z)$ the Green's function and the return function for the operator $\mathcal{A}$ on $\ell^{2}(\mathcal{X})$.

Corollary 1. (see $([7,8]$ in the group case) Let the notation be as above, and set

$$
s_{i}(w)=w\left(\phi_{1}\left(w^{-1}\right)+\cdots+\hat{\phi}_{i}\left(w^{-1}\right)+\cdots+\phi_{n}\left(w^{-1}\right)\right) .
$$

Then, for $1 \leq i \leq n$,

$$
\mathcal{G}(w)=G_{i}\left(w-s_{i}(w)\right)
$$

Proof. By the proof of Theorem 1

$$
\mathcal{F}(z)=\left(1-\phi_{1}(z)-\cdots-\phi_{n}(z)\right)^{-1}
$$


Substituting this into eq. (7), and using eq. (4), we obtain

$$
\mathcal{G}\left(\frac{1}{z}\right)=G_{i}\left(\frac{1-\phi_{1}(z)-\cdots-\hat{\phi}_{i}-\cdots-\phi_{n}(z)}{z}\right) .
$$

With $z^{-1}=w$, this implies the claim.

Remark. Specializing Corollary 1 to the case when $A_{i}$ are convolution operators on the groups $X_{i}$, and $n=2$, we obtain a known result ([7], Theorem 5.1). If $A_{i}$ are the incidence operators of Cayley graphs (here $n$ is arbitrary), then Corollary 1 (equivalently, Theorem 1) is also in the literature ([8], Theorem 4.9).

COROllary 2. Let the notation be as above, and set, for $1 \leq i \leq n$

$$
\xi_{i}(z)=\frac{1-\phi_{1}(z)-\cdots-\hat{\phi}_{i}-\cdots-\phi_{n}(z)}{z} .
$$

Then eq. (7) is equivalent to the system $(1 \leq i \leq n)$

$$
G_{i}\left(\xi_{i}(z)\right)=\frac{n-1}{\sum_{i=1}^{n} \xi_{i}(z)-z^{-1}} .
$$

The Green's function of $(\mathcal{X}, e)$ satisfies

$$
\mathcal{G}(z)=\frac{n-1}{\sum_{i=1}^{n} \xi_{i}\left(z^{-1}\right)-z} .
$$

Proof. Straightforward computation, using eq. (11).

We put Corollary 2 in a form more suitable for our applications.

Corollary 3. Let $\left(X_{i}, e_{i}\right), 1 \leq i \leq n$, be arbitrary rooted sets, let $A_{i}$ be operators on $\ell^{2}\left(X_{i}\right)$, and let $G_{i}(z)$ be their Green's functions. Let $x_{i}=x_{i}(t)$ be the solutions of the system $(1 \leq i \leq n)$

$$
G_{i}\left(x_{i}\right)=\frac{n-1}{\sum_{i=1}^{n} x_{i}(t)-t} .
$$

Then the Green's function of the free product operator, $\mathcal{A}=*_{i=1}^{n} A_{i}$, is given by

$$
\mathcal{G}(t)=\frac{n-1}{\sum_{i=1}^{n} x_{i}(t)-t} .
$$


The following is known in the group case (compare with [7], Corollary 5.2).

Corollary 4. Let $\left(X_{i}, e_{i}\right)$ be finite rooted sets, and let $A_{i}$ be (selfadjoint) operators on $\ell^{2}\left(X_{i}\right), 1 \leq i \leq n$. Then the Green's function of the free product, $*_{i=1}^{n} A_{i}$, is algebraic.

Proof. The Green's functions $G_{i}$ are rational, hence the solutions of the system (15) are algebraic functions. Eq. (16) implies the claim.

\section{$\S 3 . \quad E x p l i c i t$ Green's Functions and Spectra}

The system (15) can be explicitly solved only in special cases. We will do this for two classes of examples. As a benefit, we will completely determine the spectra of certain free product operators.

Let $G$ be a Green's function of a selfadjoint operator $L$. That is, for $t \in \mathbf{H}$, the upper half-plane, $G(t)=\langle f|(t-L)^{-1} f>$, for a certain vector $f$. Note that our definition of the resolvent, $R(t)=(t-L)^{-1}$, agrees with the one in $[4,7]$, and differs by sign from the one in [9]. The spectral measure of $L$ is determined by $\lim G(x+i \epsilon)$, as $\epsilon \rightarrow 0$. We say that a Green's function is algebraic, if its analytic continuation is an algebraic function on a Riemann surface, $\mathcal{R}$, which is a finite-sheeted branched covering of the Riemann sphere, $p: \mathcal{R} \rightarrow \mathbf{C}$. The following problem typically arises in this situation. Given $G(t)$ as a function on $\mathcal{R}$, and $r \in \mathcal{R}$, such that $p(r) \in \mathbf{R} \subset \overline{\mathbf{H}}$, determine whether $r$ belongs to the 'physical sheet' of $\mathcal{R}$. We will use a well known fact, which we formulate as a lemma, for future reference.

LEMMA 1. Let $G$ be an algebraic Green's function, and let $p: \mathcal{R} \rightarrow \mathbf{C}$ be the corresponding branched covering. Let $t \in \mathbf{R}$ be a point, which is not in the branch locus of $p$, and let $r \in \mathcal{R}$ be a point above $t$.

1. If $G$ has a pole at $r$, and $\operatorname{Res}_{r} G<0$, then $r$ is not in the physical sheet.

2. Let $G(r)$ be finite. If $G(r) \in \mathbf{R}$ and $G^{\prime}(r)>0$, then $r$ is not in the physical sheet.

\subsection{Free products of two operators}

Throughout this subsection we consider invariant selfadjoint operators $A$ and $B$ on $\ell^{2}(X)$ and $\ell^{2}(Y)$ respectively, where $X$ and $Y$ are countable (e. g., finite) sets. Then $A * B$ is an invariant selfadjoint operator on $\ell^{2}(X * Y)$. We are interested in the Green's function and the spectrum of $A * B$. The 
standing assumption will be that the spectrum of each of the operators $A, B$ is pure point, with two distinct eigenvalues.

By the preceding material, the Green's functions satisfy

$$
G_{A}(z)=\frac{u}{z-a}+\frac{v}{z-b}, G_{B}(z)=\frac{r}{z-c}+\frac{s}{z-d}
$$

where $a<b, c<d$, and $u, v, r, s>0$, see eq. (6). Set

$$
\begin{gathered}
g=(2 u-1) b+(2 v-1) a, h=(2 r-1) d+(2 s-1) c, S=a+b+c+d, \\
\Sigma=g+h, \Delta=g-h, T=(a+b)(c+d)+2(a b+c d),
\end{gathered}
$$

and define the following polynomials:

$$
\begin{gathered}
M(t)=-2 t+S, \\
N(t)=-\Sigma t^{2}+S \Sigma t-\frac{1}{2}\left[\Sigma T+g(c-d)^{2}+h(a-b)^{2}\right], \\
D(t)=(t-(a+c))(t-(a+d))(t-(b+c))(t-(b+d)), \\
E(t)=g h t^{2}-S g h t+\frac{1}{4}\left[g^{2}(c-d)^{2}+h^{2}(a-b)^{2}+2 g h T\right] .
\end{gathered}
$$

Proposition 1. Let $A$ and $B$ be operators, satisfying the standing assumptions. Let the notation be as above, and set $R(t)=D(t)+E(t)$. Then the Green's function of $A * B$ satisfies

$$
\mathcal{G}(t)=\frac{N(t)-M(t) \sqrt{R(t)}}{2 D(t)} .
$$

Proof. Set $x=x_{1}(t), y=x_{2}(t)$. By Corollary 3

$$
\frac{u}{x-a}+\frac{v}{x-b}=\frac{r}{y-c}+\frac{s}{y-d}=\frac{1}{x+y-t} \text {. }
$$

This is equivalent to the system (here we use $u+v=r+s=1$, see eq. (6))

$$
\begin{aligned}
& x y=\left(t+\frac{1}{2}(g-a-b)\right) x+\frac{1}{2}(g+a+b) y+\left(-\frac{1}{2}((g+a+b) t+a b),\right. \\
& x y=\frac{1}{2}(h+c+d) x+\left(t+\frac{1}{2}(h-c-d)\right) y+\left(-\frac{1}{2}((h+c+d) t+c d) .\right.
\end{aligned}
$$


We separate the variables to arrive to two quadratic equations on $x, y$ with polynomial (in $t$ ) coefficients. Solving them, and using eq. (16), we obtain the Green's function of $A * B$ :

$$
\mathcal{G}(t)=\frac{2\left(t+\frac{1}{2}(\Delta-S)\right)\left(-t+\frac{1}{2}(\Delta+S)\right)}{N(t)+M(t) \sqrt{R(t)}} .
$$

Multiplying the numerator and the denominator in eq. (23) by $N(t)-M(t)$. $\sqrt{R(t)}$, we get in the denominator:

$$
N^{2}(t)-M^{2}(t)[D(t)+E(t)]=\left[N^{2}(t)-M^{2}(t) E(t)\right]-M^{2}(t) D(t) .
$$

We will prove the identity

$$
N^{2}(t)-M^{2}(t) E(t)=\Delta^{2} D(t) .
$$

Set $N^{2}(t)-M^{2}(t) E(t)=X(t)=\sum_{i=0}^{4} X_{i} t^{i}$, and $D(t)=\sum_{i=0}^{4} D_{i} t^{i}$. The coefficients $X_{i}, D_{i}$ are polynomials in $a, b, c, d, g, h$, and the identity above is equivalent to the system $(0 \leq i \leq 4)$

$$
X_{i}(a, b, c, d, g, h)=\Delta^{2} D_{i}(a, b, c, d) .
$$

These identities are verified directly from eqs. (18-21). For $i=4,3$ the verification is very simple. To sketch a proof of eq. (24) for $i=2,1,0$, we will use a self-explanatory notation for the coefficients of the polynomials $N(t), M(t)$. Then, by eqs. (18-21)

$$
X_{2}=-2 \Sigma N_{0}-4 E_{0}+S^{2} \Sigma^{2}-5 S^{2} g h=-2 \Sigma N_{0}-4 E_{0}-S^{2} g h+\Delta^{2} S^{2} .
$$

By eqs. $(19,21)$

$$
-2 \Sigma N_{0}-4 E_{0}-S^{2} g h=\Delta^{2} T,
$$

hence $X_{2}=\Delta^{2}\left(S^{2}+T\right)$. By eq. (18), $D_{2}=S^{2}+T$, which proves eq. (24) for $i=2$. The identity $X_{1}=\Delta^{2} D_{1}$ is equivalent to the one we have just proved (note that $D_{1}=-S T$ ). For $i=0$ eq. (24) becomes

$$
N_{0}^{2}-S^{2} E_{0}=\Delta^{2}(a+c)(a+d)(b+c)(b+d) .
$$

This is proved directly from eqs. $(19,21)$, and we leave it to the reader.

Our identity and eq. (18) yield

$$
N^{2}(t)-M^{2}(t) R(t)=4\left(t+\frac{1}{2}(\Delta-S)\right)\left(-t+\frac{1}{2}(\Delta+S)\right) D(t)
$$

which proves the claim. 
Proposition 1 means, in particular, that the Green's function of $A * B$ is algebraic on the Riemann surface $\mathcal{R}=\left\{(t, w): w^{2}=R(t)\right\}$, which is a 2-sheeted covering of the Riemann sphere, via $p(t, w)=t$. The sheets of $\mathcal{R}$ correspond to the two branches of $\sqrt{R(t)}$, where on the physical sheet we have, asymptotically, $\sqrt{R(t)} \sim t^{2}$, as $t \rightarrow \infty$. The involution $(t, w) \mapsto(t,-w)$ interchanges the physical and the nonphysical sheets. Note that $\mathcal{R}$ is an elliptic curve, since $R$ is a quartic polynomial, and $p: \mathcal{R} \rightarrow \mathbf{C}$ is the canonical covering.

Let $\mathcal{R}_{-}$(resp. $\mathcal{R}_{+}$) be the part of the physical (resp. nonphysical) sheet above $\mathbf{H}$. Since the branch locus of $p: \mathcal{R} \rightarrow \mathbf{C}$ consists of zeros of the quartic polynomial $R$, and since $\mathcal{R}_{-}$and $\mathcal{R}_{+}$are disjoint, we conclude that $R$ has no roots in $\mathbf{H}$. Thus, all roots of $R$ are real, which also follows directly from eqs. (20-21), see the proof of Theorem 2, below.

Let the notation be as above. If the inequalities

$$
\begin{gathered}
a+d \neq S / 2, \\
E(a+c), E(b+d), E(a+d), E(b+c) \neq 0
\end{gathered}
$$

hold, we say that the operators $A, B$ are in general position.

Proposition 2. Let the operators $A$ and $B$ satisfy the standing assumptions. If they are in general position then the point spectrum of $A * B$ consists of at most two eigenvalues; One of them is contained in $\{a+c, b+d\}$, and the other in $\{a+d, b+c\}$.

Proof. By the preceding discussion and eq. (22), the poles of $\mathcal{G}$ on the Riemann surface $\mathcal{R}=\left\{(t, w): w^{2}-R(t)=0\right\}$ are contained in the set $p^{-1}(\{a+c, b+d, a+d, b+c\})$, where $p: \mathcal{R} \rightarrow \mathbf{C}$ is the canonical projection. By eqs. $(26-27),\left|p^{-1}(\{a+c, b+d, a+d, b+c\})\right|=8$. Let $t_{0}$ be any of the four numbers $a+c, a+d, b+c, b+d$, and let $p_{0}, r_{0} \in \mathcal{R}$ be the two points above it. Note that if $p_{0}=\left(t_{0}, w_{0}\right)$, then $r_{0}=\left(t_{0},-w_{0}\right)$. Since, by eq. (25)

$$
\left(N\left(t_{0}\right)-M\left(t_{0}\right) w_{0}\right)\left(N\left(t_{0}\right)+M\left(t_{0}\right) w_{0}\right)=0
$$

and

$$
N\left(t_{0}\right)+M\left(t_{0}\right) w_{0}=0, N\left(t_{0}\right)-M\left(t_{0}\right) w_{0}=2 N\left(t_{0}\right) \neq 0,
$$

the function $\mathcal{G}$ has a pole at $p_{0}$, and $r_{0}$ is a regular point. Thus, $\mathcal{G}$ has four poles in $\mathcal{R}$, one above each of the numbers $a+c, a+d, b+c, b+d$. Moreover, the poles are simple, and the residue of $\mathcal{G}$ at $p_{0}$ is $2 N\left(t_{0}\right) / D^{\prime}\left(t_{0}\right) \neq 0$. 
By straightforward computations

$$
\begin{aligned}
& N(a+c)=N(b+d)=-\frac{1}{2}(b-a+d-c)[g(d-c)+h(b-a)] \\
& N(a+d)=N(b+c)=-\frac{1}{2}(b+c-a-d)[g(c-d)+h(b-a)]
\end{aligned}
$$

and

$$
\begin{gathered}
0<D^{\prime}(b+d)=[b-a+d-c](d-c)(b-a)=-D^{\prime}(a+c) \\
D^{\prime}(b+c)=-[b+c-a-d](d-c)(b-a)=-D^{\prime}(a+d)
\end{gathered}
$$

Thus

$$
\begin{aligned}
& \frac{N(a+d)}{D^{\prime}(a+d)}=\frac{1}{2}\left[\frac{g}{b-a}-\frac{h}{d-c}\right]=-\frac{N(b+c)}{D^{\prime}(b+c)}, \\
& \frac{N(a+c)}{D^{\prime}(a+c)}=\frac{1}{2}\left[\frac{g}{b-a}+\frac{h}{d-c}\right]=-\frac{N(b+d)}{D^{\prime}(b+d)} .
\end{aligned}
$$

Next, we invoke Lemma 1. By eq. (28) (resp. eq. (29)), at most one of the two poles of $\mathcal{G}$ above $a+d, b+c$ (resp. above $a+c, b+d$ ) is in the physical sheet.

A complete analysis of the point spectrum of $A * B$ requires detailed calculations. We will return to this and related questions elsewhere [5]. Next we turn to the continuous spectrum of $A * B$.

Proposition 3. Let the operators $A$ and $B$ satisfy the standing assumptions (we don't assume they are in general position), and let the notation be as above. Then $A * B$ has no singular continuous spectrum: $\sigma_{c}(A * B)=\sigma_{a c}(A * B)$. If $t_{1}<t_{2}<t_{3}<t_{4}$ are the roots of the quartic polynomial $R=D+E$, then $\sigma_{c}(A * B)=\left[t_{1}, t_{2}\right] \cup\left[t_{3}, t_{4}\right]$.

Proof. Set $L=A * B, Z=X * Y$. Then $L$ is an invariant selfadjoint operator on $\ell^{2}(Z)$. Let $\mathcal{G}_{z}(t), z \in Z, t \in \mathbf{H}$ be the Green's function of $L$, corresponding to $\delta_{x} \in \ell^{2}(Z)[4]$. By invariance of $L$, we have $\mathcal{G}_{z}(t)=\mathcal{G}_{e}(t)$, where $e$ is the root of $Z$. Let $\mu$ be the spectral measure, corresponding to $\delta_{e}$, and let $\mu_{f}$ be the one corresponding to $f \in \ell^{2}(Z)$ [4]. Then, by invariance of $L$, the measure $\mu_{f}$ is absolutely continuous with respect to $\mu$ [7], hence $\mu$ is the spectral measure of $L$.

Let $\mu=\mu_{a c}+\mu_{s c}+\mu_{p p}$ be the standard decomposition of $\mu$. Using that on the physical sheet $\mathcal{G}_{e}=\mathcal{G}$, which is algebraic, by eq. (22), and the standard 
characterization of the singular continuous spectrum, [9] Section 1.1, we obtain that $\mu_{s c}=0$. Thus $\mu=\mu_{a c}+\mu_{p p}$.

Let $t=x+i \epsilon$, and for any function on $\mathbf{H}$ set $f(x+i 0)=\lim _{\epsilon \rightarrow 0} f(x+i \epsilon)$, if the limit exists. Then (see [9], Theorem 1.6)

$$
\pi d \mu_{a c}(x)=|\Im \mathcal{G}(x+i 0)| d x .
$$

Eq. (22) implies the claim.

Remark. The fact that $A * B$ has no singular continuous spectrum, probably, is quite general. For instance, $\mu_{s c}(A * B)=0$ if $A$ and $B$ are arbitrary (right) convolution operators on discrete groups [7].

LEMMA 2. Let $A$ and $B$ be a pair of operators, satisfying the standing assumptions, and let the notation be as above. Set

$$
\begin{gathered}
\alpha=\frac{1}{4}(b+d-a-c)^{2}+\frac{1}{4}(a+d-b-c)^{2}-g h, \\
\gamma=(a-b)^{2}(c-d)^{2}+g^{2} h^{2}-g^{2}(c-d)^{2}-h^{2}(a-b)^{2} .
\end{gathered}
$$

Then $0<\sqrt{\gamma} \leq \alpha$. The equality $\alpha=\sqrt{\gamma}$ holds if and only if $b-a=d-c$ and $u=r, v=s$ (see eq. (17) for notation).

Proof. Substituting the expressions for $g$ and $h$ in terms of the spectral data of $A, B$ into the formulas above, we obtain

$$
\begin{gathered}
2 \alpha=[(a-b)-(c-d)]^{2}+2[(b-a)(d-c)[1-(u-v)(r-s)] \\
\gamma=(a-b)^{2}(c-d)^{2}\left[1-(u-v)^{2}\right]\left[1-(r-s)^{2}\right]
\end{gathered}
$$

Since $|u-v|,|r-s|<1$, we have

$$
0<[(a-b)-(c-d)]^{2}<2 \alpha<[(a-b)+(c-d)]^{2},
$$

and $\gamma>0$. The inequality $\alpha \geq \sqrt{\gamma}$ follows from

$$
[1-(u-v)(r-s)]^{2} \geq\left[1-(u-v)^{2}\right]\left[1-(r-s)^{2}\right]
$$

The equality $\alpha=\sqrt{\gamma}$ holds if and only if $(a-b)-(c-d)=0$ and $u-v=r-s$. Since $u+v=r+s=1$, the latter equation is equivalent to $u=r$. 
Recall that $S=a+b+c+d$, and set

$$
\beta=\frac{1}{4}(g-h)\left[g(c-d)^{2}-h(a-b)^{2}\right]+\frac{1}{16}(b+d-a-c)^{2}(a+d-b-c)^{2} \text {. }
$$

LEMma 3. Let the setting be as above. Then the quartic polynomial $R$ (see Proposition 1) satisfies

$$
R(w+S / 2)=w^{4}-\alpha w^{2}+\beta .
$$

Proof. By eqs. (20-21), $D(t)$ and $E(t)$ are invariant with respect to $t \rightarrow S-t$. Hence $R(w+S / 2)$ is an even polynomial in $w$. The claim follows by a direct computation.

Definition 3. Let $A_{i}$ be bounded linear operators on Hilbert saces $\mathcal{H}_{i}, i=1,2$. They are essentially unitarily equivalent if there exist Hilbert spaces $\mathcal{M}_{1}, \mathcal{M}_{2}$ such that the operators $A_{i} \otimes I d_{\mathcal{M}_{i}}$ on $\mathcal{H}_{i} \otimes \mathcal{M}_{i}, i=1,2$ are unitarily equivalent.

THEOREM 2. Let the operators $A$ and $B$ satisfy the standing assumptions, and let the notation be as above.

1) Suppose that $A$ and $B-\lambda I d$ are not essentially unitarily equivalent for any $\lambda$. Then the continuous spectrum of $A * B$ is a union of two disjoint intervals:

$$
\begin{aligned}
\sigma_{c}(A * B) & =\left[\frac{S}{2}-\sqrt{\frac{1}{2}(\alpha+\sqrt{\gamma})}, \frac{S}{2}-\sqrt{\frac{1}{2}(\alpha-\sqrt{\gamma})}\right] \\
& \cup\left[\frac{S}{2}+\sqrt{\frac{1}{2}(\alpha-\sqrt{\gamma})}, \frac{S}{2}+\sqrt{\frac{1}{2}(\alpha+\sqrt{\gamma})}\right] .
\end{aligned}
$$

2) If there exists $\lambda$ such that $A$ and $B-\lambda I d$ are essentially unitarily equivalent, then the continuous spectrum of $A * B$ is a single interval:

$$
\sigma_{c}(A * B)=\left[\frac{S}{2}-\sqrt{\alpha}, \frac{S}{2}+\sqrt{\alpha}\right] .
$$

Proof. By Lemma 2 and Lemma 3, all roots of $R$ are real. They are

$$
\begin{aligned}
\frac{S}{2}-\sqrt{\frac{1}{2}(\alpha+\sqrt{\gamma})} & <\frac{S}{2}-\sqrt{\frac{1}{2}(\alpha-\sqrt{\gamma})} \\
& \leq \frac{S}{2}+\sqrt{\frac{1}{2}(\alpha-\sqrt{\gamma})} \\
& <\frac{S}{2}+\sqrt{\frac{1}{2}(\alpha+\sqrt{\gamma})}
\end{aligned}
$$


If $\alpha>\sqrt{\gamma}$, then the roots are distinct, and Proposition 3 applies. If $\alpha=\sqrt{\gamma}$, then

$$
\sqrt{R(t)}=(t-S / 2) \sqrt{(t-S / 2+\sqrt{\alpha})(t-S / 2-\sqrt{\alpha})} .
$$

The argument of Proposition 3 applies and shows that $\sigma_{c}(A * B)=[S / 2-$ $\sqrt{\alpha}, S / 2+\sqrt{\alpha}]$.

It remains to show that the dichotomy $\alpha>\sqrt{\gamma}$ versus $\alpha=\sqrt{\gamma}$ is equivalent to the dichotomy of the Theorem. By Lemma 2, $\alpha=\sqrt{\gamma}$ if and only if there exist $p, q>0, p+q=1$, and $\lambda \in \mathbf{R}$ such that

$$
G_{A}(z)=\frac{p}{z-a}+\frac{q}{z-b}, G_{B}(z)=\frac{p}{z-a-\lambda}+\frac{q}{z-b-\lambda} .
$$

Equivalently, the eigenvalues of $A$ (resp. $B$ ) are $a<b$ (resp. $a+\lambda<b+\lambda$ ), with the same relative multiplicities $p$ and $q$. Let $m, n$ be natural numbers such that $m|X|=n|Y|=h$. Setting $\mathcal{M}=\mathbf{C}^{m}, \mathcal{N}=\mathbf{C}^{n}$, we obtain that $A \otimes I d_{\mathcal{M}}$ and $(B-\lambda) \otimes I d_{\mathcal{N}}$ are selfadjoint operators, acting on the same space $\mathbf{C}^{h}$, with the same eigenvalues $a<b$, of the same multiplicities. Hence, they are unitarily equivalent. We leave the proof of the opposite implication to the reader.

Using eqs. (30-31) we can directly express the intervals comprising the continuous spectrum of $A * B$ in terms of the spectral data of the operators $A$ and $B$. Leaving the general case to the reader, we will restict ourselves to the case when $\sigma_{c}(A * B)$ is a single interval. The following is an immediate corollary of Theorem 2 and its proof.

COROLlary 5. Let the operators $A$ and $B$ satisfy the standing assumptions, and let the notation be as above. Then the set $\sigma_{c}(A * B)$ is connected if and only if $A$ and $B$ are essentially unitarily equivalent.

Let this be the case, and let $a, b, \lambda, p, q$ be as in eq. (33). Then

$\sigma_{c}(A * B)=[a+b+\lambda-2(b-a) \sqrt{p q}, a+b+\lambda+2(b-a) \sqrt{p q}] \subset[2 a+\lambda, 2 b+\lambda]$.

The inclusion is strict (on both ends), unless $p=q=1 / 2$.

\subsection{Free powers of an operator}

In this subsection we consider arbitrary 'free powers' of a single operator $A$ on $\ell^{2}(X)$, where $(X, e)$ is a rooted set. Let $G(t)$ be the Green's function of $A$, based at $e$. For $n>1$ we denote by $*^{n} A$ the $n$-th free power of $A$, i.e., $*^{n} A=*_{i=1}^{n} A_{i}$, where $A_{i}=A$ for all $i$. 
Proposition 4. Let $\mathcal{G}_{n}(t)$ be the Green's function of $*^{n} A, n>1$. Then

$$
\mathcal{G}_{n}(t)=\frac{n-1}{n x-t}
$$

where $x=x(t)$ satisfies

$$
G(x)=\frac{n-1}{n x-t}
$$

Proof. This is a special case of Corollary 3, where we have, by symmetry, $x_{i}=x_{j}$ for all $i, j$.

From now until the end of Section 3 the standing assumption will be that $|X|<\infty$ and that $A$ is invariant and selfadjoint. In addition, we assume that $A$ has two eigenvalues. Thus $G(z)=p /(z-a)+q /(z-b)$, where $a<b$ and $p, q>0, p+q=1$. Some of the propositions to follow remain valid, with obvious modifications, if $|X|=\infty$.

Proposition 5. Let $A$ satisfy the standing assumptions, and let the notation be as above. Set

$$
\begin{aligned}
P_{n}(t) & =(n-2) t+n^{2}(p b+q a)-n(n-1)(a+b) \\
R_{n}(t) & =t^{2}+2[(n-2)(p b+q a)-(n-1)(a+b)] t \\
& +[n(p b+q a)-(n-1)(a+b)]^{2}+4(n-1) a b .
\end{aligned}
$$

Then the Green's function of $*^{n} A$ satisfies

$$
\frac{1}{2} \mathcal{G}_{n}(t)=\frac{n \sqrt{R_{n}(t)}-P_{n}(t)}{(t-n a)(t-n b)} .
$$

Proof. Specializing in eq. (35), we have

$$
\frac{p}{x-a}+\frac{q}{x-b}=\frac{n-1}{n x-t}
$$

which gives a quadratic equation on $x$. Substituting $x(t)$ into eq. (34) and getting rid of the radicals in the denominator, like in the proof of Proposition 1 , we obtain the claim. 
Proposition 5 means, in particular, that the Green's function $\mathcal{G}_{n}$ of $*^{n} A$ is algebraic on the Riemann surface $\mathcal{R}_{n}=\left\{(t, w): w^{2}=R_{n}(t)\right\}$, which is a 2 -sheeted covering of the Riemann sphere, via $p_{n}(t, w)=t$. The sheets of $\mathcal{R}$ correspond to the two branches of $\sqrt{R_{n}(t)}$, where on the physical sheet we have, asymptotically, $\sqrt{R_{n}(t)} \sim t$, as $t \rightarrow \infty$. Our considerations involving $\mathcal{G}_{n}$ as a meromorphic function on the Riemann surface $\mathcal{R}_{n}$ are analogous to those of Section 3.1, concerning the Green's function of $A * B$. If anything, they are simpler in the present case. Note that $\mathcal{R}_{n}$ is the Riemann sphere, since $R_{n}$ is a quadratic polynomial.

THEOREM 3. Let $A$ be an operator satisfying the standing assumptions, and let $G(z)=p /(z-a)+q /(z-b)$ be its Green's function. We consider the operators $*^{n} A, n>1$, and assume that $n p, n q \neq 1$ for any $n$.

1. The operator $*^{n} A$ has no singular continuous spectrum. Its absolutely continuous spectrum is a single interval:

$$
\begin{aligned}
\sigma_{a c}\left(*^{n} A\right)= & \sigma_{c}\left(*^{n} A\right) \\
= & {[(1+p(n-2)) a+(1+q(n-2)) b-2(b-a) \sqrt{(n-1) p q},} \\
& (1+p(n-2)) a+(1+q(n-2)) b+2(b-a) \sqrt{(n-1) p q}] .
\end{aligned}
$$

2. Let $p<q$ (resp. $q<p)$. Then the point spectrum of $*^{n} A$ consists of the single point $n b$ (resp. na), as long as $n<1 / p$ (resp. $n<1 / q)$. The point spectrum is empty for $n>1 / p$ (resp. $n>1 / q$ ).

Proof. 1. The proof is analogous to the argument of Theorem 2 and Proposition 3, and we leave details to the reader. The endpoints of $\sigma_{a c}\left(*^{n} A\right)$ are the two roots of $R_{n}$.

2. The argument follows the proof of Proposition 2. By eq. (36), the only possible poles of $\mathcal{G}_{n}$ in $\mathcal{R}_{n}$ are above $n a, n b \in \mathbf{R}$. Denote by $t$ any of the two numbers. A direct computation gives

$$
P_{n}(n a)=n(n q-1)(a-b), P_{n}(n b)=n(n p-1)(b-a) .
$$

Hence, $t$ is not in the branch locus, and exactly one of the two points of $\mathcal{R}_{n}$ above $t$ is a pole of $\mathcal{G}_{n}$. Let $p(t) \in \mathcal{R}_{n}$ be the pole, and let $r(t) \in \mathcal{R}_{n}$ be the regular point (we suppress the dependence on $n$, to simplify the notation). Both poles are simple, and from eqs. (36-37)

$$
\operatorname{Res}_{p(a)} \mathcal{G}_{n}=1-n q, \operatorname{Res}_{p(b)} \mathcal{G}_{n}=1-n p .
$$


A direct computation gives

$$
\mathcal{G}_{n}^{\prime}(p(a))=c(a)(1-n q), \mathcal{G}_{n}^{\prime}(p(b))=c(b)(1-n p)
$$

where $c(a), c(b)>0$. Hence the sign of $\mathcal{G}^{\prime}$ at the regular point above $a$ (resp. $b$ ) is the same as the sign of $1-n q$ (resp. $1-n p$ ). Lemma 1 implies that $p(a)$ (resp. $p(b)$ ) is in the physical sheet if and only if $1-n q>0$ (resp. $1-n p>0)$. Since $n p+n q=n \geq 2$, both $p(a)$ and $p(b)$ cannot be in the physical sheet, which implies the claim.

\section{$\S 4$. Applications}

\subsection{Graph spectra}

By the spectrum of a graph, $\Gamma$, we mean the spectrum of its incidence operator, $A_{\Gamma}$. Another set, frequently associated with $\Gamma$, is the spectrum of the graph Laplacean (see, e. g, [2]). If $\Gamma$ is regular (every vertex has the same number of edges), the two spectra are related by a translation. We will consider only symmetric (therefore regular) graphs.

If $\Gamma_{1}, \Gamma_{2}$ are two rooted graphs, we denote by $\Gamma_{1} * \Gamma_{2}$ their free product. By the construction of Section 2, this is a special case of the free product of rooted sets. If $A_{1}, A_{2}$ are the incidence operators, then $A_{1} * A_{2}$ is the incidence operator of $\Gamma_{1} * \Gamma_{2}$. If $\Gamma_{1}, \Gamma_{2}$ are symmetric graphs, i.e. there are groups, $H_{1}, H_{2}$, acting transitively on $\Gamma_{1}, \Gamma_{2}$ by automorphisms, then $H_{1} * H_{2}$ transitively acts on $\Gamma_{1} * \Gamma_{2}$, thus $\Gamma_{1} * \Gamma_{2}$ is symmetric. In particular, $\Gamma_{1} * \Gamma_{2}$ does not depend on the choice of roots in $\Gamma_{1}, \Gamma_{2}$. Everything we said so far about the free product of two graphs extends to the free products of any number of graphs. We will use the self-explanatory notation: $*_{i=1}^{n} \Gamma_{i}$, $*^{n} \Gamma$.

We denote by $K_{n}, n \geq 1$, the complete graph on $n+1$ vertices (any two vertices are neighbors). It is symmetric (under the natural action of $S_{n+1}$, the symmetric group).

THEOREM 4. Let $1 \leq m<n$. The (absolutely) continuous spectrum of $K_{m} * K_{n}$ is the union of two disjoint intervals:

$$
\begin{gathered}
I_{m, n}=\frac{1}{2}\left[m+n-2-\sqrt{4(\sqrt{ } m+\sqrt{ } n)^{2}+(m-n)^{2}}\right. \\
\left.m+n-2-\sqrt{4(\sqrt{ } m-\sqrt{ } n)^{2}+(m-n)^{2}}\right]
\end{gathered}
$$


and

$$
\begin{aligned}
& J_{m, n}=\frac{1}{2}\left[m+n-2+\sqrt{4(\sqrt{ } m-\sqrt{ } n)^{2}+(m-n)^{2}}\right. \\
&\left.m+n-2+\sqrt{4(\sqrt{ } m+\sqrt{ } n)^{2}+(m-n)^{2}}\right] .
\end{aligned}
$$

The point spectrum of $K_{m} * K_{n}$ is the set $\{-2, m-1\}$.

Proof. The spectrum of $K_{n}$ consists of two points: -1 and $n$. The multiplicities are $n$ and 1 respectively. We denote by $A, B$ the incidence operators of $K_{m}, K_{n}$, and use the notation of Section 3.1. Thus $a=1, b=$ $m, c=-1, d=n, u=m /(m+1), v=1 /(m+1), r=n /(n+1), s=1 /(n+1)$. Substituting this into eqs. (18-22), we obtain

$$
\begin{aligned}
N(t)=-(m+n-2) t^{2}+(m+n-2)^{2} t \\
-[(m+n+2)(m n-7)+24],
\end{aligned}
$$

$$
\begin{aligned}
& R_{m, n}(t) \\
=\left(t-\frac{m+n}{2}+1\right)^{4}-\left[2(m+n)+\frac{(m-n)^{2}}{2}\right]\left(t-\frac{m+n}{2}+1\right)^{2} & +\frac{1}{16}(m-n)^{2}\left[(m+n+4)^{2}-4 m n\right],
\end{aligned}
$$

and for the Green's function of the graph $K_{m} * K_{n}$ :

$$
\mathcal{G}_{m, n}(t)=\frac{N(t)+(2 t-m-n+2) \sqrt{R_{m, n}(t)}}{2(t+2)(t+m+n)(t-m+1)(t-n+1)} .
$$

We use Theorem 2 to find the absolutely continuous spectrum of $K_{m} *$ $K_{n}$. By eq. (39), $\alpha>0, \alpha^{2}-4 \beta>0$, and $\beta>0$ (because $m<n$ ). Thus, by Theorem 2, the continuous spectrum of $K_{m} * K_{n}$ is a union of two intervals, whose endpoints are $\frac{1}{2}(m+n)-1 \pm \sqrt{(\sqrt{ } m \pm \sqrt{ } n)^{2}+\left(\frac{1}{2}(m-n)\right)^{2}}$.

The inequalities eqs. (26-27) hold (because $m<n$ ), thus $A$ and $B$ are in general position. We use Proposition 2 and explicit calculations, like in the proof of Theorem 3, to compute the point spectrum of $K_{m} * K_{n}$.

EXAmples. 1 (see $[7,8]$ ). Let $m=1, n=2$. From eq. (40) we obtain the Green's function of $K_{1} * K_{2}$ :

$$
\mathcal{G}_{1,2}(t)=-t^{2}+\frac{t+1+(2 t-1) \sqrt{t^{4}-2 t^{3}-5 t^{2}-6 t+1}}{2(t+2)(t(t-1)(t+3)}
$$


By Theorem 4, the point spectrum of $K_{1} * K_{2}$ is $\{-2,0\}$, and the continuous spectrum:

$$
\begin{aligned}
{\left[\frac{1}{2}-\frac{1}{2} \sqrt{13+8 \sqrt{ } 2}, \frac{1}{2}-\frac{1}{2} \sqrt{13-8 \sqrt{ } 2}\right] } \\
\cup\left[\frac{1}{2}+\frac{1}{2} \sqrt{13-8 \sqrt{ } 2}, \frac{1}{2}+\frac{1}{2} \sqrt{13+8 \sqrt{ } 2}\right]
\end{aligned}
$$

2. Set $m=1, n>1$. By Theorem 4 , the point spectrum is $\{-2,0\}$, and $\sigma_{c}\left(K_{1} * K_{n}\right)=I_{n} \cup J_{n}$, where

$$
\begin{aligned}
& I_{n}=\left[\frac{n-1}{2}-\frac{1}{2} \sqrt{(n+1)^{2}+4+8 \sqrt{ } n}, \frac{n-1}{2}-\frac{1}{2} \sqrt{(n+1)^{2}+4-8 \sqrt{ } n}\right], \\
& J_{n}=\left[\frac{n-1}{2}+\frac{1}{2} \sqrt{(n+1)^{2}+4-8 \sqrt{ } n}, \frac{n-1}{2}+\frac{1}{2} \sqrt{(n+1)^{2}+4+8 \sqrt{ } n}\right] .
\end{aligned}
$$

THEOREM 5. Let $K_{r}, r \geq 1$, be the complete graph on $r+1$ vertices, and let $\mathcal{G}_{n}^{(r)}$ be the Green's function of $*^{n} K_{r}, n>1$. Then

$$
\mathcal{G}_{n}^{(r)}=\frac{(n-2) t+n(r-1)-n \sqrt{t^{2}-2(r-1) t+(r+1)^{2}-4 n r}}{2(t+n)(t-n r)} .
$$

For $r>1$ the point spectrum of $*^{n} K_{r}$ is a single point, $\{-n\}$, if $2 \leq n \leq r$, and is empty if $n>r$. The graph $*^{n} K_{1}$ has no point spectrum. The (absolutely) continuous spectrum of $*^{n} K_{r}$ :

$$
\sigma_{c}\left(*^{n} K_{r}\right)=[r-1-2 \sqrt{(n-1) r}, r-1+2 \sqrt{(n-1) r}] .
$$

Proof. Straightforward from Proposition 5 and Theorem 3.

EXAMPLES. 1. The graph $*^{n+1} K_{1}$ is the $n+1$-regular tree, $T_{n}$. Specializing to $r=1$ in Theorem 5, we obtain its Green's function:

$$
G_{T_{n}}(t)=\frac{(n-1) t-(n+1) \sqrt{t^{2}-4 n}}{2(t+n+1)(t-n-1)}
$$

and the absolutely continuous spectrum: $\sigma\left(T_{n}\right)=[-2 \sqrt{n}, 2 \sqrt{n}]$ (there is no point spectrum). These results are well known (see, e.g., [2]). 2. Setting $r=2, n=2$ in Theorem 5, we obtain: $\sigma_{c}\left(*^{2} K_{2}\right)=[1-2 \sqrt{2}, 1+2 \sqrt{2}]$, and $\sigma_{p}\left(*^{2} K_{2}\right)=\{-2\}$. We leave details to the reader. 


\subsection{Random walks on free products}

A random walk on a graph, $\Gamma$, is given by the probabilities, $0 \leq p(e) \leq$ 1 , on the edges of $\Gamma$, so that $\sum_{e \sim x} p(e)=1$ for any vertex $x \in \Gamma$. Equivalently, a random walk is determined by its transition operator, $(P f)(x)=$ $\sum_{y \sim x} P(x, y) f(y)$, and $P(x, y)=p(e)$, where $e$ is the edge joining $x$ with $y$. We say that a random walk on $\Gamma$ is invariant if its transition operator is invariant (under a transitive group on $\Gamma$ ). By the spectrum (Green's function) of a random walk on $\Gamma$ we mean the spectrum (Green's function) of its transition operator.

An invariant random walk on a rooted graph, $(\Gamma, e)$, is determined by the probabilities of the edges of $e$. For the simple random walk these probabilities are equal to $1 / d$, where $d$ is the number of edges of $e$. Let $\Gamma_{i}, 1 \leq i \leq n$, be symmetric graphs, and let $P_{i}$ be the transition operator for an invariant random walk on $\Gamma_{i}, 1 \leq i \leq n$. Any $n$-tuple, $p_{i}>0, \sum_{i=1}^{n} p_{i}=1$, defines a product random walk on $*_{i=1}^{n} \Gamma_{i}$, its transition operator is $*_{i=1}^{n} p_{i} P_{i}$. If the random walks on $\Gamma_{i}$ are simple for $1 \leq i \leq n$, and $p_{1}=\cdots=p_{n}=1 / n$, then the product random walk on $*_{i=1}^{n} \Gamma_{i}$ is also simple.

THEOREM 6. ([3]) Let $r \geq 1, n>1$. The (absolutely) continuous spectrum of the simple random walk on $*^{n} K_{r}$ is $\frac{1}{r n}[r-1-2 \sqrt{(n-1) r}, r-1+$ $2 \sqrt{(n-1) r}$. The point spectrum is nonempty if and only if $2 \leq n \leq r$. Then it consists of a single point, $\{-1 / r\}$.

Proof. The transition operator of the simple random walk on a $k$ regular graph, $\Gamma$, is $k^{-1} A_{\Gamma}$. In our case, $k=n r$, and the spectrum of $A_{\Gamma}$ is given by Theorem 5 .

THEOREM 7. Let $1 \leq m \leq n$, and $p, q>0, p+q=1$. We assume that $(p, m) \neq(q, n)$, and consider the product random walk on $K_{m} * K_{n}$ defined by these data. Its (absolutely) continuous spectrum is the union of two intervals, $I, J$ (depending on $p, q, m, n)$. Define $\ell_{ \pm}(p, q, m, n)>0$ by

$$
\ell_{ \pm}^{2}=\frac{1}{4}(p-q)^{2}+\frac{1}{4}\left(\frac{p}{m}-\frac{q}{n}\right)^{2}+\frac{1}{2}\left[\frac{p}{m}+\frac{q}{n} \pm \frac{4 p q}{\sqrt{m n}}\right] .
$$

Then

$$
\begin{aligned}
& I=\frac{1}{2}\left[1-\left(\frac{p}{m}+\frac{q}{n}\right)-2 \ell_{+}, 1-\left(\frac{p}{m}+\frac{q}{n}\right)-2 \ell_{-}\right] \\
& J=\frac{1}{2}\left[1-\left(\frac{p}{m}+\frac{q}{n}\right)+2 \ell_{-}, 1-\left(\frac{p}{m}+\frac{q}{n}\right)+2 \ell_{+}\right] .
\end{aligned}
$$


If $m<n$, the point spectrum is $\{-(p / m+q / n), p-q / n\}$. If $m=n>1$, the point spectrum is $\{-1 / m\}$. If $m=n=1$, the point spectrum is empty.

Proof. The transition operator of this random walk is the free product $m^{-1} p A_{m} * n^{-1} q A_{n}$, where $A_{\ell}$ is the incidence matrix of the complete graph $K_{\ell}$. In the notation of Section 3.1, $a=-p / m, b=p, c=-q / n, d=q, u=$ $m /(m+1), v=n /(n+1), r=n /(n+1), s=1 /(n+1)$. We compute the parameters $\alpha$ and $\gamma$ of Theorem 2 , and obtain

$m^{2} n^{2}[\alpha \pm \sqrt{\gamma}]=\frac{1}{2} m^{2} n^{2}(p-q)^{2}+\frac{1}{2}(p n-q m)^{2}+m n[p n+q m \pm 4 p q \sqrt{m n}]$.

It is elementary to check that $p n+q m \pm 4 p q \sqrt{m n} \geq 0$, hence the expression above is positive (we have ruled out $p=q, m=n$ ). Thus $\ell_{ \pm}=$ $\sqrt{(\alpha \pm \sqrt{ } \gamma) / 2}>0$, and Theorem 2 gives the continuous spectrum.

We use Proposition 2 and explicit calculations, like in the proof of Theorem 3, to determine the point spectrum.

Remark. Theorems 6 and 7 together yield the spectra of all product random walks on $K_{m} * K_{n}$. Proposition 1 allows to find explicitly their Green's functions. We leave this to the reader.

\section{REFERENCES}

[1] K. Aomoto and Y. Kato, Green functions and spectra on free products of cyclic groups, Ann. Inst. Fourier, 38 (1988), 59-85.

[2] R. Brooks, The spectral geometry of k-regular graphs, J. Analyse Math., 57 (1991), 120-151.

[3] D. I. Cartwright and P. M. Soardi, Random walks on free products, quotients and amalgams, Nagoya Math. J., 102 (1986), 163-180.

[4] N. Danford and J. T. Schwartz, Linear Operators, Interscience, New York, 1963.

[5] E. Gutkin, Point spectra of free products of operators, in preparation.

[6] G. Kuhn, Random walks on free products, Ann. Inst. Fourier, 4 (1991), 467-491.

[7] J. C. McLaughlin, Random walks and convolution operators on free products, Doctoral Dissertation, New York University, 1986.

[8] G. Quenell, Combinatorics on free product graphs, Geometry of the Spectrum, pp. 257-282.

[9] B. Simon, Spectral analysis of rank one perturbations and applications, CRM Proceedings and Lecture Notes, pp. 109-149.

[10] D. Voiculescu, Addition of certain noncommuting random variables, J. Funct. Anal., 66 (1986), 323-346. 
[11] W. Woess, Nearest neighbor random walks on free products of discrete groups, Boll. U.M.I., 5-B (1986), 961-982.

[12] W. Woess, Random walks on infinite graphs and groups - a survey of selected topics, Bull. London Math. Soc., 26 (1994), 1-60.

\author{
Mathematics Department \\ University of Southern California \\ Los Angeles, CA 90089-1113 \\ U.S.A. \\ egutkin@math.usc.edu
}

